\title{
Gauss-Bonnet gravity renders negative tension braneworlds unstable
}

\author{
Christos Charmousis and Jean-François Dufaux \\ LPT, Université de Paris-Sud, Bât. 210, 91405 Orsay CEDEX, France
}

\begin{abstract}
We show that the addition of the Gauss-Bonnet term to Einstein gravity induces a tachyon mode in the spin 2 fluctuations of the Randall-Sundrum I model. We demonstrate that this instability is generically related to the presence of a flat negative tension brane, of co-dimension one, embedded in an anti-de Sitter background. In particular its presence is independent of $Z_{2}$-symmetry or compactness of the extra dimension. The gravitational tachyon mode, persists for arbitrarily small but non vanishing Gauss-Bonnet coupling. It is a bound state localised on the negative tension brane, much like the graviton zero-mode is localised on a positive tension one. We discuss the possible resolution of this instability by the inclusion of induced gravity terms on the branes or by an effective four-dimensional cosmological constant.
\end{abstract}

PACS numbers: 04.50.+h, 11.25.Db, 11.25.Mj

Negative tension branes appear quite naturally as endpoints of spacetime in higher than 4 dimensions. For example they may appear in non-oriented string theories as orientifold planes [1], 2], or in orbifold compactifications of braneworld models. In the Randall-Sundrum [3] model (RS1), their embedding in anti-de Sitter spacetime provides an elegant resolution of the hierarchy problem, once the interbrane distance is fixed [4]. Interestingly, any realisation of such a geometrical hierarchy in string theory seems to also require the presence of negative tension objects [5], orientifolds.

Higher order curvature corrections also appear in string theory as $\alpha^{\prime}$ corrections in low energy effective actions 6]. The Gauss-Bonnet term in particular, appears (for example) in heterotic string theory rendering the low energy effective action ghostfree [7] around a flat background. More importantly, in a non-perturbative approach, one obtains such a curvature invariant from purely geometric considerations in higher than 4 dimensions. Indeed the Gauss-Bonnet combination is the unique higher order curvature term, in five or six dimensions, leading to a classical gravity theory which satisfies the physical assumptions of General Relativity in four dimensions [8]. In particular, the resulting field equations involve only up to second order derivatives of the metric. This is an essential assumption when treating backgrounds with boundaries, and it renders spacetime perturbations wavelike.

In this letter we consider braneworld models involving a negative tension brane, with four-dimensional Poincaré invariance, embedded in five-dimensional anti-de Sitter (adS) background. We will show that the Gauss-Bonnet term induces generically at least one tachyon mode in the spin 2 fluctuations of such models.

Consider a model with two branes in Einstein-Gauss-Bonnet (EGB) gravity :

$$
S=\frac{M^{3}}{2} \int d^{5} x \sqrt{-g}\left(-2 \Lambda+R+\alpha\left[R^{2}-4 R_{a b} R^{a b}+R_{a b c d} R^{a b c d}\right]\right)-\int d^{4} x \sqrt{-\gamma_{1}} T_{1}-\int d^{4} x \sqrt{-\gamma_{2}} T_{2}
$$

We will take $\alpha \geq 0$ in the following as in the case of string slope expansion $[\underline{6}$. The solution with a smooth (Einstein) $\alpha \rightarrow 0$ limit and four-dimensional Poincare invariance reads,

$$
d s^{2}=e^{2 A(y)} \eta_{\mu \nu} d x^{\mu} d x^{\nu}+d y^{2}
$$

where

$$
A(y)=-k y \quad ; \quad k=\sqrt{\frac{1}{4 \alpha}\left(1-\sqrt{1+\frac{4}{3} \alpha \Lambda}\right)}
$$

The solution is defined for $4 \alpha k^{2} \leq 1$. The two flat branes have tension $T_{1}$ and $T_{2}$, and are located at $y=0$ and $y=y_{c}$ respectively. We also assume $Z_{2}$-symmetry accross the corresponding orbifold fixed points. The junction conditions [9] then require,

$$
\frac{T_{1}}{M^{3}}=-\frac{T_{2}}{M^{3}}=2 k\left(3-4 \alpha k^{2}\right)
$$

where $T_{1}>0$ and $T_{2}<0$. This setup is just the generalisation, in Gauss-Bonnet gravity, of the RS 1 model [3] where typically, $e^{k y_{c}} \sim 10^{15}$. 
Let us now consider the spin 2 fluctuations around this background. For these it is sufficient to consider a linear perturbation in Gaussian normal gauge,

$$
d s^{2}=e^{2 A(y)}\left(\eta_{\mu \nu}+h_{\mu \nu}^{(m)}(x) \psi_{m}(y)\right)+d y^{2}
$$

with $h_{\mu \nu}^{(m)}(x)$ transverse and tracefree. The metric fluctuations verify, $\square_{(4)} h_{\mu \nu}^{(m)}(x)=m^{2} h_{\mu \nu}^{(m)}(x)$, leading to plane wave separation of variables, $h_{\mu \nu}^{(m)}(x)=\epsilon_{\mu \nu} e^{i p_{\lambda} x^{\lambda}}$ with 4-dimensional momenta $p^{2}=-m^{2}$, where $\epsilon_{\mu \nu}$ is the constant polarisation tensor. We do not consider here the scalar mode describing the interbrane distance [10], the radion, which will not be relevant for our purpose [20]. The perturbation equation in the bulk reads,

$$
-\left(p(y) \psi_{m}^{\prime}(y)\right)^{\prime}=m^{2} w(y) \psi_{m}
$$

where a prime denotes derivative with respect to $y$ and

$$
p(y)=e^{4 A}\left(1-4 \alpha A^{\prime 2}\right), \quad w(y)=e^{2 A}\left(1-4 \alpha A^{\prime 2}-4 \alpha A^{\prime \prime}\right)
$$

Because of the Gauss-Bonnet term, $p$ and $w$ now involve derivatives in the background field $A(y)$, which are not continuous in the presence of branes. For example, $w=\hat{w}+\left[-4 \alpha e^{2 A} A^{\prime}\right] \delta\left(y-y_{i}\right)$, where $\hat{w}$ stands for the continuous part of $w$, and [ ] denotes the jump across the brane location $y=y_{i}$ (see also for example [11]). For the two brane system we consider here, we have in the bulk $\left(0<y<y_{c}\right)$,

$$
p(y)=\left(1-4 \alpha k^{2}\right) e^{-4 k y}, \quad \hat{w}(y)=\left(1-4 \alpha k^{2}\right) e^{-2 k y}
$$

while the junction conditions resulting from (6) read:

$$
\begin{array}{r}
\psi_{m}^{\prime}\left(0^{+}\right)=-\frac{4 \alpha k}{1-4 \alpha k^{2}} m^{2} \psi_{m}(0) \\
\psi_{m}^{\prime}\left(y_{c}^{-}\right)=-\frac{4 \alpha k}{1-4 \alpha k^{2}} m^{2} e^{2 k y_{c}} \psi_{m}\left(y_{c}\right)
\end{array}
$$

For $\alpha=0$ (or $m=0$ ), these are usual Neumann boundary conditions. In Gauss-Bonnet gravity however, we have mixed boundary conditions involving the energies of the modes themselves. As a result, their norm $\left\|\psi_{m}\right\|$ has to include suitable boundary terms (see also [12]),

$$
0 \leq \int_{0}^{y_{c}} d y p \psi_{m}^{\prime 2}=m^{2}\left[\int_{0}^{y_{c}} d y \hat{w} \psi_{m}^{2}+4 \alpha k\left(\psi_{m}^{2}(0)-e^{-2 k y_{c}} \psi_{m}^{2}\left(y_{c}\right)\right)\right]=m^{2}\left\|\psi_{m}\right\|^{2}
$$

where the first equality results from multiplying (6) by $\psi_{m}$, integrating by parts on $y$ and using (9)-(10). As usual, $p$ and $\hat{w}$ (8) are positive definite from (3). Therefore, for $\alpha=0$, (11) implies that $m^{2}$ has to be non negative. However, this standard positivity argument breaks down if any of the boundary terms in (11) is negative as in the case of negative tension branes. Tachyonic states may then appear in the spectrum. Note also that the norm is negative in that case. In this sense any tachyon in the bulk perturbations can be also interpreted as a ghost at the level of the 4-dimensional effective action. Indeed expanding (1) to second order we obtain,

$$
S_{e f f} \sim-\frac{M^{3}}{4}\left\|\psi_{m}\right\|^{2} \int d^{4} x\left(\nabla h_{\mu \nu}\right)^{2}+m^{2} h_{\mu \nu} h^{\mu \nu}
$$

For $m^{2}<0$ in (11) there is always a wrong relative sign between the kinetic and mass terms which signals classical instability.

We now show that such a tachyon state is indeed present for the two brane system. Any solution of (6) with $m^{2}=-\mu^{2}<0$ is of the form,

$$
\psi_{\mu}(y)=e^{2 k y}\left(A_{\mu} I_{2}\left(\frac{\mu}{k} e^{k y}\right)+B_{\mu} K_{2}\left(\frac{\mu}{k} e^{k y}\right)\right)
$$

with $A_{\mu}$ and $B_{\mu}$ real constants. A linear perturbation containing such an eigenmode will grow exponentially with time and will signal the classical instability of the background. A tachyon mode of mass $\mu$ exists if on imposing (9)-(10) on (13), $x=\frac{\mu}{k}>0$, is solution of (for $\mu \neq 0$ ),

$$
f(x)=g(x)
$$


with

$$
\begin{aligned}
& f(x)=\left(I_{1}(\chi x)-\zeta \chi x I_{2}(\chi x)\right)\left(K_{1}(x)+\zeta x K_{2}(x)\right) \\
& g(x)=\left(I_{1}(x)-\zeta x I_{2}(x)\right)\left(K_{1}(\chi x)+\zeta \chi x K_{2}(\chi x)\right)
\end{aligned}
$$

We have introduced two numerical parameters:

$$
\zeta:=\frac{4 \alpha k^{2}}{1-4 \alpha k^{2}} \quad \text { and } \quad \chi:=e^{k y_{c}}>1
$$

The parameter $\chi$ describes the interbrane distance and is typically a large number. Parameter $\zeta$ describes deviation from General Relativity and we have $0 \leq \zeta<+\infty$. For $\alpha \Lambda$ small, which essentially means dominant Einstein gravity, $\zeta$ is also small, with $\zeta=0$ for $\alpha=0$. Alternatively $\zeta$ goes to infinity for $\alpha \Lambda=-4 / 3$. Apart from these limiting cases, it is easy to show that (14) always admits a finite non-vanishing solution for $x$. The functions $f(x)$ and $g(x)$ are shown in Fig. 1]
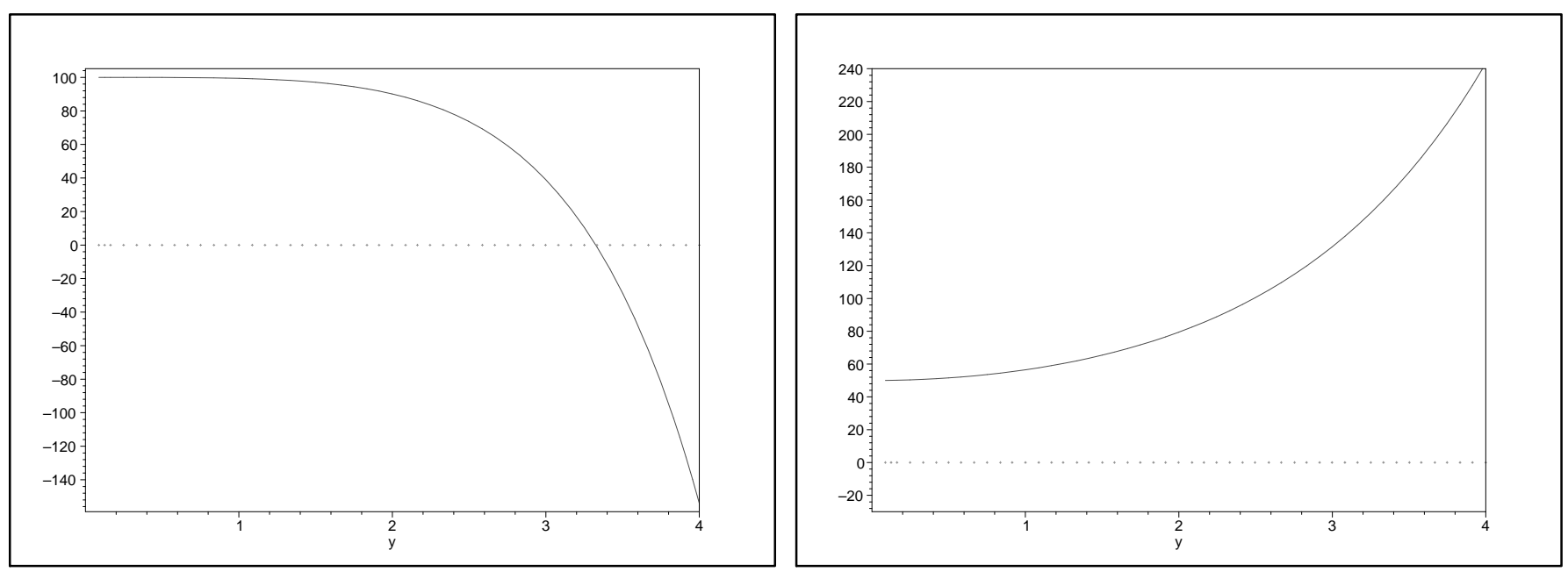

FIG. 1: $f$ (line) and $g$ (dots) as functions of $\chi x$ for $\zeta=0.5$ (left) and $\zeta=0$ (right) ; $\chi=100$ here.

For $x \rightarrow 0$,

$$
f(x) \rightarrow\left(\zeta+\frac{1}{2}\right) \chi, \quad g(x) \rightarrow \frac{\left(\zeta+\frac{1}{2}\right)}{\chi}
$$

whereas as $x \rightarrow+\infty$,

$$
f(x) \sim-\frac{1}{2} \zeta^{2} \sqrt{\chi} x e^{(\chi-1) x}, \quad g(x) \sim-\frac{1}{2} \zeta^{2} \sqrt{\chi} x e^{-(\chi-1) x}
$$

Therefore $f(x)>g(x)>0$ for $x \simeq 0$, while for $x \rightarrow+\infty g(x) \rightarrow 0$ and $f(x) \rightarrow-\infty$ (note however that when $\zeta=0$, $f(x) \rightarrow+\infty$ as $x \rightarrow+\infty)$. Since $f(x)$ and $g(x)$ are continuous on $] 0+\infty$, they have to intersect at least once at finite $x$, corresponding to the tachyon mass. The instability persists for arbitrarily small but non vanishing coupling $\alpha$.

In order to illustrate the qualitative properties of the tachyon mode, we go now to the analog quantum mechanical picture. Consider $|z|=\frac{1}{k}\left(e^{k|y|}-1\right)$, with the branes now located at $z=0$ and $z=z_{c}$, and define $\Phi(z)=e^{-3 k|y| / 2} \psi(y)$. Then $\Phi(z)$ obeys the Schrödinger equation,

$$
-\frac{d^{2} \Phi}{d z^{2}}+V(z) \Phi=m^{2}\left(1+\frac{2 \zeta}{k} \delta(z)-\frac{2 \zeta \chi}{k} \delta\left(z-z_{c}\right)\right) \Phi
$$


Note the presence of the mass-dependent distributional terms in the right-hand-side. For the bound state solutions $m^{2}=-\mu^{2} \leq 0$, the effective potential reads,

$$
V_{\mathrm{eff}}^{\mu}=\frac{15 k^{2}}{4(k|z|+1)^{2}}-\left(3 k-2 \frac{\zeta}{k} \mu^{2}\right) \delta(z)+\left(3 \frac{k}{\chi}-2 \frac{\chi}{k} \zeta \mu^{2}\right) \delta\left(z-z_{c}\right)
$$

where we have included the energy-dependent distributional terms for the sake of illustration. Much may be understood from the distributional contributions to the potential (20), which are either attractive if their overall coefficient is negative, or repulsive if it is positive. The contribution centered on the Planck brane at $z=0$ is attractive for $\mu=0$, and it allows for the usual normalisable bound state zero mode. However, we see that the contribution at $z=z_{c}$ may now be attractive for $\mu \neq 0$. Namely the negative tension brane can support a new normalisable tachyonic bound state if $\zeta \neq 0$. The wave function of the two bound states is shown in Fig. 2]

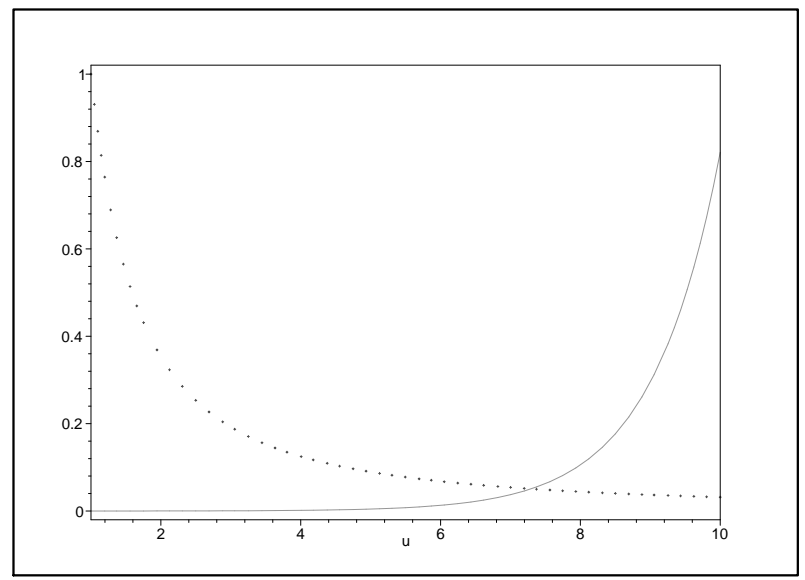

FIG. 2: Tachyon mode $\Phi_{\mu}(z)$ (line) localised on the TeV brane and zero mode $\Phi_{0}(z)$ (dotted) localised on the Planck brane, for the two branes system $(\chi=10$ and $\zeta=0.1)$

We may approximate the mass and the wave function of the tachyon $\Phi_{\mu}$ by solving (19) in the vicinity of the negative tension brane at $z=z_{c}$. Then, for $\chi>>1$, the bulk potential (20) is approximately constant and we can neglect the boundary term at $z=0$. To constant order in $\zeta$, the tachyon mass is,

$$
\mu \simeq \frac{k}{\chi \zeta}(1+3 \zeta)
$$

The distributional term at $z=z_{c}$ in (20) is then indeed attractive. The corresponding wave function is localised on the negative tension brane, much like the zero mode is bound on the positive tension one. If one is to stabilize the hierarchy between the $T e V$ scale and the four-dimensional Planck mass $M_{\mathrm{Pl}}$ as in [3] then we have to live on the negative tension brane where the physical masses are measured as $m_{c}=\chi m$ and $k \sim T e V$. The approximation (21) for the tachyon mass $\mu$ holds in particular in this case, and it gives $\mu_{c} \sim T e V$ if $\zeta \sim 1$, whereas $\mu_{c} \sim M_{\mathrm{Pl}}$ if $\zeta$ is fine-tuned to zero at the $10^{-15}$ level. Generically, the smaller the coupling $\alpha$, the larger the tachyon mass and the more localised its wave function: Even when the Gauss-Bonnet coupling $\alpha$ is small, the Gauss-Bonnet term in the action does not act as a perturbative correction. In particular, the limit $\alpha \rightarrow 0$ is discontinuous for the tachyon mass, namely we have then $\mu \rightarrow \infty$, whereas there is no tachyon when $\alpha=0$ exactly.

The instability of negative tension branes is independent of $Z_{2}$ symmetry or compactness of the extra dimension. Consider a single $Z_{2}$-symmetric positive tension brane in an infinite extra dimension, ie. the second Randall-Sundrum model [13], in EGB gravity. Any tachyon mode would still be given by (13), but the boundary condition at infinity now requires $A_{\mu}=0$. Imposing the junction condition (9) at $y=0$, we end up with the second factor of $f(x)$ in (15) vanishing, which has no solution. This model has therefore stable spin 2 fluctuations. Accordingly, sending the negative tension brane to infinity in (21), ie. $\chi \rightarrow \infty$, sets $\mu=0$. On the contrary if we choose to keep the negative tension brane, we have $B_{\mu}=0$ at infinity and we loose the zero mode keeping the tachyonic one. Another illustration is provided by the Gauss-Bonnet version of the Lykken-Randall model 14], where the tension of the second brane may be either positive or negative. In this setup we keep $Z_{2}$-symmetry across a first brane at $y=0$, but break it across a second brane at $y=y_{c}$. For $|y|<y_{c}$, the bulk cosmological constant is still denoted as $\Lambda$, and the background 
solution is still given by (3). However, one introduces now another cosmological constant $\tilde{\Lambda}$ in the bulk for $|y|>y_{c}$, with corresponding warp factor $\tilde{k}$ in the background solution. Then, the tension of the first brane is still given by $T_{1}$ in (4), while for the second brane it reads, $T_{2}=M^{3}(\tilde{k}-k)\left[3-4 \alpha k^{2}-4 \alpha \tilde{k}^{2}\right]$ which is positive or negative, according to the sign of $(\tilde{k}-k)$. The candidate tachyon mode, is given seperately in two regions (13), and as before we impose (14) with similar asymptotic behaviour, except that we now have,

$$
f(x) \sim \frac{1}{2} \zeta^{2} \sqrt{\chi} x e^{(\chi-1) x}\left(\frac{\tilde{k}}{k}-1\right) \quad \text { for } x \rightarrow+\infty
$$

Hence, when both branes have positive tension, that is when $\tilde{k}>k, f(x) \rightarrow+\infty$ for $x \rightarrow+\infty$, rather than minus infinity (18), and there is no tachyon. In the contrary if one of the branes has negative tension, we have tachyonic instability. It is therefore clear that in anti-de Sitter background, any inclusion of a flat negative tension brane destabilises the system in EGB gravity.

The presence of induced gravity terms on the branes can modify the above conclusions (see also [15]). Indeed, consider the action:

$$
S_{t}=S+\frac{M^{3}}{2}\left(\beta_{1} \int_{y=0} d^{4} x \sqrt{-\gamma} R^{(4)}[\gamma]+\beta_{2} \int_{y=y_{c}} d^{4} x \sqrt{-\gamma} R^{(4)}[\gamma]\right)
$$

Only the junction conditions for the massive modes are modified. We can follow the same analysis as above defining,

$$
\zeta_{1}=\frac{4 \alpha k^{2}+\beta_{1} k / 2}{1-4 \alpha k^{2}} \text { and } \zeta_{2}=\frac{4 \alpha k^{2}-\beta_{2} k / 2}{1-4 \alpha k^{2}}
$$

For simplicity we neglect terms of order $\frac{1}{\chi^{2}}$. Requiring the effective four-dimenssional Planck mass squared to be positive then gives $\zeta_{1} \geq-1 / 2$. Furthermore, the presence of brane induced gravity terms on the negative tension brane may lead to the radion field being a ghost (see for instance [16]). This also signals instability, although at the quantum level this time. Generalising the method used in 17] to the action (23), we find that for this not to be the case, the parameters have to satisfy $\left(1-4 \alpha k^{2}\right) /\left(1+2 \zeta_{2}\right) \geq 0$. Taking these constraints into account, the range of parameters for which the gravitational tachyon mode may be avoided reduces to [21]

$$
\zeta_{1} \geq 0 \text { and }-\frac{1}{2} \leq \zeta_{2} \leq 0
$$

To conclude we have shown that any negative tension brane in an adS background induces a bound state tachyon mode in the context of Einstein-Gauss-Bonnet gravity. It would be interesting to study if the instability portrayed here persists for other spacetime geometries as well (see also [12] where Gauss-Bonnet gravity destabilises scalar field brane solutions). In string theory both Gauss-Bonnet corrections [6] and negative tension boundaries, orientifold planes [1], 2] can appear. A typical example is that of open type I $S O(32)$ string theory. Orientifolds there appear as non-dynamical 8-branes of RR charge [19] and negative tension. The Gauss-Bonnet term also appears at the level of the disk since the underlying theory is $\mathrm{S}$ dual to the $S O(32)$ heterotic string (where the Gauss-Bonnet term appears at tree-level [6]). Clearly the field content is richer and much more complex in this case, especially since the Gauss-Bonnet term would be coupled to a varying dilaton field. Furthermore even if the instability persisted as a bound state to the orientifold, then the effective action approach could be unjustified. The stability issues for string theory backgrounds certainly require further careful investigation.

A final issue is the endpoint of the instability. One can argue that the brane action may pick up induced gravity terms (at the price of adding 2 more coupling constants to the original action) and in this case we showed that one may avoid the instability by restricting the according couplings (25). Alternatively the RS I solution is not a stable vacuum of the action (1) and therefore one may question what the true vacuum of such a theory is. For instance had we considered 4 dimensional dS or adS branes would the spin 2 instability persist ? Such a question on the stable vacuum may be interesting if stringent constraints are required for the effective 4 dimensional cosmological constant. This is a question we hope to be addressing in the near future.

We are particularly grateful to Pierre Binetruy and Jihad Mourad for their encouragement and enlightening discussions. We also thank Stephen Davis and Valery Rubakov for discussions at the early stages of this work. CC thanks Luigi Pilo for discussions on mixed boundary conditions.

[1] A. Sagnotti, published in Cargese Summer Inst.1987:0521-528 (QC174.45:N2:1987), arXiv:hep-th/0208020 J. Polchinski and Y. Cai, Nucl. Phys. B 296, 91 (1988). 
[2] J. Polchinski, String Theory, Vol. I and II. J. Polchinski, Phys. Rev. Lett. 75, 4724 (1995).

[3] L. Randall and R. Sundrum, Phys. Rev. Lett. 83, 3370 (1999).

[4] E.g.: W. D. Goldberger and M. B. Wise, Phys. Rev. Lett. 83, 4922 (1999). J. Martin, G. N. Felder, A. V. Frolov, M. Peloso and L. Kofman, arXiv:hep-th/0309001 J. Lesgourgues and L. Sorbo, arXiv:hep-th/0310007

[5] E.g.: S. B. Giddings, S. Kachru and J. Polchinski, Phys. Rev. D 66, 106006 (2002).

[6] D. J. Gross and J. H. Sloan, Nucl. Phys. B 291 (1987) 41. R. R. Metsaev and A. A. Tseytlin, Nucl. Phys. B 293 (1987) 385.

[7] B. Zwiebach, Phys. Lett. B 156 (1985) 315.

[8] D. Lovelock, J. Math. Phys. 12 (1971) 498.

[9] S. C. Davis, Phys. Rev. D 67, 024030 (2003). E. Gravanis and S. Willison, Phys. Lett. B 562, 118 (2003).

[10] C. Charmousis, R. Gregory and V. A. Rubakov, Phys. Rev. D 62, 067505 (2000).

[11] Y. M. Cho, I. P. Neupane and P. S. Wesson, Nucl. Phys. B 621 (2002) 388. K. A. Meissner and M. Olechowski, Phys. Rev. D 65 (2002) 064017.

[12] C. Charmousis, S. C. Davis and J. F. Dufaux, JHEP 0312, 029 (2003).

[13] L. Randall and R. Sundrum, Phys. Rev. Lett. 83 (1999) 4690.

[14] J. Lykken and L. Randall, JHEP 0006, 014 (2000).

[15] H. Davoudiasl, J. L. Hewett and T. G. Rizzo, JHEP 0308, 034 (2003) S. L. Dubovsky and M. V. Libanov, JHEP 0311, 038 (2003). G. Kofinas, R. Maartens and E. Papantonopoulos, JHEP 0310, 066 (2003).

[16] C. Csaki, M. L. Graesser and G. D. Kribs, Phys. Rev. D 63, 065002 (2001)

[17] L. Pilo, R. Rattazzi and A. Zaffaroni, JHEP 0007 (2000) 056

[18] Y. Shtanov and A. Viznyuk, arXiv:hep-th/0312261

[19] J. Polchinski and E. Witten, Nucl. Phys. B 460, 525 (1996).

[20] a direct calculation in the lines of [10] shows that the radion mode remains a massless scalar in Gauss-Bonnet gravity

[21] We thank Y.Shtanov for pointing out a previous mistake in [25], which was in contradiction with a result in [18]. 